\title{
ANÁLISIS DE DIFERENCIAS EN EL USO, CONOCIMIENTO Y ACTITUDES HACIA LAS NUEVAS TECNOLOGÍAS ENTRE PERSONAS CON Y SIN ENFERMEDAD MENTAL GRAVE
}

\author{
Carla Natalia Casiano Yanicelli \\ María del Carmen Lozano Segura \\ Cristian Aguilera Ruiz \\ Ana Manzano León \\ Universidad de Almería \\ carlacasiano@hotmail.es
}

https://doi.org/10.17060/ijodaep.2017.n1.v4.1050

Fecha de Recepción: 1 Marzo 2017

Fecha de Admisión: 1 Abril 2017

\section{RESUMEN}

En este artículo se comparan personas con y sin enfermedad mental grave respecto al uso, conocimiento, interés y actitudes hacia las nuevas tecnologías de la información (TIC). En el estudio han participado siete personas con enfermedad mental grave y siete sin enfermedad mental. Los resultados muestran que existen diferencias significativas intergrupos en relación al uso, conocimiento, interés y actitudes hacia las nuevas tecnologías de la información.

Palabras clave: enfermedad mental grave, nuevas tecnologías, esquizofrenia, internet.

\section{ABSTRACT}

Analysis of differences regard to use, knowledge, interest and attitudes towards new information technologies between people with and without severe mental disorders

This article compares people with severe mental disorders and people without mental disorders regard to use, knowledge, interest and attitudes towards new information technologies (ICT). In the study participated seven persons with severe mental illness, and seven persons without mental illness. The results show that there are significant intergroup differences in relation to the use, knowledge, interest and attitudes toward new information technologies.

Key words: severe mental illness, new technologies, schizophrenia, internet.

\section{INTRODUCCIÓN}

Según la Organización Mundial de la Salud (OMS): «La salud es un estado de completo bienestar físico, mental y social, y no solamente la ausencia de afecciones o enfermedades». El concepto de salud mental se define como un estado de bienestar en el cual el individuo es consciente de sus 


\section{ANÁLISIS DE DIFERENCIAS EN EL USO, CONOCIMIENTO Y ACTITUDES HACIA LAS NUEVAS TECNOLOGÍAS ENTRE PERSONAS CON Y SIN ENFERMEDAD MENTAL GRAVE}

propias capacidades, puede afrontar las tensiones normales de la vida cotidiana, puede trabajar de forma productiva y es capaz de hacer una contribución a su comunidad.

Por otro lado, las enfermedades mentales son condiciones médicas que interrumpen el pensamiento, los sentimientos, el humor, la habilidad de relacionarse con otros y el funcionamiento diario de una persona. Así, los trastornos mentales conllevan la pérdida del bienestar psicosocial, un deterioro en el ámbito laboral o académico y alteraciones en las actividades y relaciones sociales. Entre las enfermedades mentales graves (EMG) se encuentran las siguientes: depresión, esquizofrenia, trastorno bipolar, trastorno obsesivo-compulsivo y trastorno de la personalidad, entre otras (Bravo, 2007).

En las personas que sufren EMG son comunes las discapacidades crónicas en las funciones cognitivas, los sentimientos de aislamiento y soledad y la dificultad para realizar de manera adecuada el autocuidado. Todos estos déficits repercuten negativa y significativamente en las esferas laboral, económica, familiar, social y psicológica, generando en ocasiones la búsqueda de apoyo social (Välimäk, et al. 2012).

Tras el diagnóstico de enfermedad mental, los pacientes sufren el rechazo por parte de la sociedad relacionado con sus síntomas. Con lo cual, no sólo deben enfrentarse a las propias dificultades asociadas a la enfermedad, sino también a las que se originan a partir del estigma y la discriminación (Bravo, 2007).

Según Brogna (2005), reseñado en Bravo (2007), la exclusión social se considera como un proceso de desintegración social que conlleva una ruptura de la relación entre individuo y sociedad, siendo el estigma un factor de exclusión social.

Actualmente, las "Tecnologías de la Información y la Comunicación" (TIC) son una de las principales bases de la comunicación universal. Las TIC han pasado a ocupar una gran parte en la vida cotidiana de las personas, aportando elementos positivos como: acceso a información y diversión, favorecer las relaciones sociales e incentivar la comunicación.

No obstante, el incremento de información en formato digital conlleva una mayor brecha digital y separación de la sociedad de quienes no utilizan las TIC en su vida diaria (Moya, 2009). Las personas con enfermedad mental crónica a menudo sufren fracasos en el acceso a la información y en la comunicación. Teniendo en cuenta la totalidad de personas con algún tipo de discapacidad, ya sea física o psicológica, las personas con enfermedad mental son el segundo grupo con la tasa más baja de uso del ordenador. Según Huang y Su (2009) en Taiwán, el porcentaje de personas con enfermedad mental que utiliza el ordenador $(22,6 \%)$ es incluso más bajo que el de personas con discapacidad visual que lo utiliza $(23,1 \%)$ (Huang \& Su 2009).

A partir de lo anterior, el objetivo de la presente investigación es comprobar si existen diferencias entre las personas con y sin problemas de salud mental en cuanto al conocimiento, opiniones y uso de nuevas tecnologías.

\section{MÉTODO}

\section{Participantes}

En este estudio han participado catorce personas de ambos sexos. Los participantes han sido agrupados según su estado de salud mental, así, se formaron dos grupos de siete individuos cada uno (cinco hombres y dos mujeres):

Personas diagnosticadas de enfermedad mental grave: dentro de este grupo las edades están comprendidas entre los 28 y 49 años, con una edad media de 37,14, y una desviación típica de 7,57. Los integrantes son usuarios de la Asociación El Timón de El Ejido y todos poseen un diagnóstico de enfermedad mental grave, en su mayoría esquizofrenia. 
Personas sin diagnóstico de enfermedad mental: compuesto por personas que nunca han sido diagnosticadas de trastorno mental, con edades comprendidas entre los 30 y los 51 años, y con una edad media de 39,86 y una desviación típica de 7,99.

Los participantes han sido seleccionados teniendo en cuenta la disponibilidad de los mismos y la accesibilidad. Además, se ha controlado que los sujetos de ambos grupos fueran similares en cuanto a edad, sexo y nivel de estudios.

\section{Procedimiento}

El procedimiento consistió en administrar a ambos grupos de sujetos un cuestionario dirigido a evaluar el conocimiento, el uso y las opiniones acerca de las nuevas tecnologías. Una vez recabados Ios datos, se procedió a realizar el análisis estadístico mediante el programa SPSS.

\section{Instrumentos}

Los participantes fueron evaluados utilizando dos cuestionarios elaborados ad hoc, referentes al conocimiento, uso y opinión acerca de las nuevas tecnologías.

\section{RESULTADOS}

A continuación, se mencionarán los resultados obtenidos tras analizar mediante la prueba $U$ de Mann-Whitney las respuestas de los grupos de personas sin enfermedad mental y con enfermedad mental.

Se encontraron diferencias estadísticamente significativas entre los grupos con y sin enfermedad mental al preguntarles si sabían qué son las nuevas tecnologías de la información ( $p=0.023)$. Concretamente, el total de las personas sin enfermedad mental (siete) respondió sía esta cuestión, mientras que tan solo tres personas con enfermedad mental respondieron de manera afirmativa y cuatro lo hicieron de forma negativa. En cambio, no se encontraron diferencias significativas al preguntar si sabían qué son el correo electrónico, los buscadores y las redes sociales (Tabla 1).

Tabla 1.

Conocimientos acerca de las nuevas tecnologías por parte de sujetos con enfermedad mental grave (EMG) y sin enfermedad mental.

\begin{tabular}{|l|c|c|c|c|}
\hline \multicolumn{5}{|c|}{ CONOCIMIENTOS } \\
\hline \multirow{2}{*}{ Ítem } & \multicolumn{2}{c|}{ CON EM } & \multicolumn{2}{c|}{ SIN EM } \\
\hline & SI & NO & SI & NO \\
\hline 1. TIC & 3 & 4 & 7 & 0 \\
\hline 6. Correo e. & 5 & 2 & 7 & 0 \\
\hline 10. Buscador & 5 & 2 & 7 & 0 \\
\hline 14. Redes sociales & 4 & 3 & 7 & 0 \\
\hline
\end{tabular}

Cuando se preguntó a los sujetos si sabían usar el correo electrónico se encontró que, dentro del grupo sin enfermedad mental cinco respondieron mucho y dos algo. En cambio, en el grupo con enfermedad mental, cuatro respondieron nada, dos algo y uno mucho. Estas diferencias resultaron ser estadísticamente significativas ( $p=0.014)$ (Tabla 2).

En las preguntas dirigidas a averiguar si sabían usar los buscadores y las redes sociales, también se obtuvieron diferencias significativas entre los dos grupos $(p=0.008$ y $p=0.001$, respectivamente). Concretamente, al preguntarles por los buscadores, seis personas respondieron mucho y 
una algo dentro del grupo de personas sin enfermedad mental, mientras que de las personas con enfermedad mental tres respondieron nada, tres algo y una mucho; al preguntarles por las redes sociales todos los individuos sin enfermedad mental respondieron mucho, al contrario, en el grupo con enfermedad mental cinco respondieron nada y dos algo (Tabla 2).

También se encontraron diferencias significativas entre los dos grupos en las siguientes preguntas: ¿Le gustaría aprender a usar el correo electrónico? $(p=0.014)$; ¿Le gustaría aprender a usar los buscadores? $(p=0.020)$, ¿Considera que es útil para usted el medio internet $(p=0.023)$. A la primera de estas preguntas dos personas sin enfermedad mental contestaron algo y cinco nada. En la segunda, un individuo respondió algo y seis poco. Por último, a la tercera de estas preguntas, todas (siete) las personas sin enfermedad mental contestaron mucho. Por el contrario, en el grupo de personas con enfermedad mental, cuatro respondieron mucho, dos algo y una nada en la primera pregunta; cinco contestaron mucho, una algo y una nada a la segunda; y finalmente, tres personas contestaron mucho y cuatro algo a la tercera pregunta (Tabla 2).

Tabla 2.

Conocimientos, intereses y actitudes acerca de las nuevas tecnologías por parte de sujetos con enfermedad mental grave y sin enfermedad mental.

\begin{tabular}{|l|c|c|c|c|c|c|c|c|c|}
\hline \multicolumn{1}{|c|}{ CONOCIMIENTOS, INTERESES Y ACTITUDES } \\
\hline \multirow{2}{*}{ Ítem } & \multicolumn{1}{|c|}{ MUCHO } & \multicolumn{2}{c|}{ ALGO } & \multicolumn{2}{c|}{ POCO } & \multicolumn{2}{c|}{ NADA } \\
\cline { 2 - 10 } & CON & SIN & CON & SIN & CON & SIN & CON & SIN \\
\hline 4. Aprender TIC & 4 & 3 & 2 & 4 & 0 & 0 & 1 & 0 \\
\hline 7. Sabe usar correo e. & 1 & 5 & 2 & 2 & 0 & 0 & 4 & 0 \\
\hline 9. Aprender correo e. & 4 & 0 & 2 & 2 & 0 & 0 & 1 & 5 \\
\hline 11. Sabe usar buscador & 1 & 6 & 3 & 1 & 0 & 0 & 3 & 0 \\
\hline 13. Aprender buscador & 5 & 0 & 1 & 1 & 0 & 6 & 1 & 0 \\
\hline 15. Sabe usar redes soc. & 0 & 7 & 2 & 0 & 0 & 0 & 5 & 0 \\
\hline 17. Aprender redes soc. & 2 & 3 & 4 & 4 & 0 & 0 & 1 & 0 \\
\hline 18. Información & 6 & 7 & 1 & 0 & 0 & 0 & 0 & 0 \\
\hline 19. Interacción y com. & 5 & 6 & 2 & 1 & 0 & 0 & 0 & 0 \\
\hline 20. Integración & 4 & 5 & 1 & 1 & 1 & 1 & 1 & 0 \\
\hline 21. Aprender cosas & 5 & 7 & 2 & 0 & 0 & 0 & 0 & 0 \\
\hline 22. Participación soc. & 3 & 4 & 4 & 1 & 0 & 1 & 0 & 1 \\
\hline 23. Entretenerse & 6 & 7 & 1 & 0 & 0 & 0 & 0 & 0 \\
\hline 24. Útil internet & 3 & 7 & 4 & 0 & 0 & 0 & 0 & 0 \\
\hline 25. Interés en internet & 5 & 7 & 2 & 0 & 0 & 0 & 0 & 0 \\
\hline 26. Mejorar RR.SS & 4 & 2 & 1 & 2 & 2 & 3 & 0 & 0 \\
\hline 27. Mejorar ánimo & 3 & 0 & 3 & 7 & 0 & 0 & 1 & 0 \\
\hline
\end{tabular}

En cuanto a la disponibilidad de TIC, se obtuvieron diferencias significativas entre ambos grupos para las siguientes tecnologías: cámara digital $(p=0.023)$ mp3 $(p=0.023)$, ordenador $(p=0.023)$, GPS $(p=0.037)$, internet $(p=0.023)$ y otros $(p=0.010)$. Sin embargo, no hubo diferencias en cuanto al acceso a televisor y teléfono móvil (Tabla 3).

Asimismo, se encontraron diferencias estadísticamente significativas respecto al uso de MP3 $(p=0.023)$, ordenador $(p=0.007)$, GPS $(p=0.007)$, Internet $(p=0.007)$ y otros $(p=0.037)$ (Tabla 3$)$. 
Tabla 3.

Disponibilidad de nuevas tecnologías por parte de sujetos con enfermedad mental grave y sin enfermedad mental.

\begin{tabular}{|c|c|c|c|c|c|c|c|c|c|c|c|c|c|c|c|c|}
\hline \multicolumn{17}{|c|}{ DISPONIBILIDAD DE TICS } \\
\hline Respuestas & \multicolumn{2}{|c|}{ Móvil } & \multicolumn{2}{|c|}{ Cámara dig. } & \multicolumn{2}{|c|}{ MP3 } & \multicolumn{2}{|c|}{ Ordenador } & \multicolumn{2}{|c|}{ GPS } & \multicolumn{2}{|c|}{ Televisor } & \multicolumn{2}{|c|}{ Internet } & \multicolumn{2}{|c|}{ Otros } \\
\hline ítem & CON & SIN & CON & SIN & CON & SIN & CON & SIN & CON & SIN & CON & SIN & CON & SIN & CON & SIN \\
\hline 2. TIC casa & 5 & 7 & 3 & 7 & 3 & 7 & 3 & 7 & 1 & 5 & 7 & 7 & 3 & $\mathrm{~T}$ & 1 & 6 \\
\hline 3. TIC que usa & 5 & 7 & 2 & 5 & 0 & 4 & 2 & 7 & 0 & 5 & 6 & 7 & 2 & 7 & 1 & 4 \\
\hline
\end{tabular}

Dentro del grupo de personas sin enfermedad mental, la mayoría declara utilizar internet, el correo electrónico, los buscadores y las redes sociales todos los días. Sin embargo, en el grupo de personas con enfermedad mental, al preguntarles por la frecuencia con que usan dichos servicios, la mayoría responde nunca o una vez al mes, y tan sólo un usuario dice utilizarlos todos los días. Tras analizar las respuestas, se encontraron diferencias estadísticamente significativas en la frecuencia de uso de internet $(p=0.003)$, correo electrónico $(p=0.005)$, buscadores $(p=0.003)$, y redes sociales $(p=0.001)$ entre el grupo de personas con enfermedad mental y el grupo sin enfermedad mental (Tabla 4).

Tabla 4.

Frecuencia de uso de nuevas tecnologías en sujetos con enfermedad mental grave y sin enfermedad mental.

\begin{tabular}{|l|c|c|c|c|c|c|c|c|}
\hline \multicolumn{10}{|c|}{ FRECUENCIA DE USO } \\
\hline \multirow{2}{*}{ ítem } & Respuestas & Todos los días & 2-3 veces/semana & Una vez al mes & \multicolumn{2}{c|}{ Nunca } \\
\cline { 2 - 10 } & CON & SIN & CON & SIN & CON & SIN & CON & SIN \\
\hline 5. Internet & 1 & 7 & 0 & 0 & 3 & 0 & 3 & 0 \\
\hline 8. Correo e. & 1 & 6 & 0 & 1 & 2 & 0 & 4 & 0 \\
\hline 12. Buscador & 1 & 7 & 0 & 0 & 3 & 0 & 3 & 0 \\
\hline 16. Redes soc. & 0 & 7 & 1 & 0 & 1 & 0 & 5 & 0 \\
\hline
\end{tabular}

Finalmente, no se encontraron diferencias estadísticamente significativas al preguntar a ambos grupo por sus opiniones acerca de las nuevas tecnologías. En este caso, las preguntas al colectivo con enfermedad mental grave son aplicadas tras haber recibido un taller sobre el uso de TIC, ya que antes del mismo resultaba imposible debido a la falta de experiencia (Tabla 5).

Tabla 5.

Opiniones acerca de las nuevas tecnologías por parte de sujetos con enfermedad mental grave (EMG) y sin enfermedad mental.

\begin{tabular}{|l|c|c|c|c|}
\hline \multicolumn{5}{|c|}{ OPINIONES } \\
\hline \multirow{2}{*}{ Respuestas } & \multicolumn{2}{|c|}{ CON EMG } & \multicolumn{2}{c|}{ SIN EM } \\
\cline { 2 - 6 } & SI & NO & SI & NO \\
\hline 28. Más cercanía & 5 & 2 & - & - \\
\hline 29. Fácil hablar por internet & 4 & 3 & 2 & 5 \\
\hline 30. Más integrado en soc. & 5 & 2 & 6 & 1 \\
\hline 31. Beneficioso internet & 6 & 1 & 7 & 0 \\
\hline 32. Entretenerte/divertirte & 7 & 0 & 7 & 0 \\
\hline 33. Menos problemas & 6 & 1 & 7 & 0 \\
\hline 34. Mejor opinión de ti & 7 & 0 & 7 & 0 \\
\hline
\end{tabular}




\section{ANÁLISIS DE DIFERENCIAS EN EL USO, CONOCIMIENTO Y ACTITUDES HACIA LAS NUEVAS TECNOLOGÍAS ENTRE PERSONAS CON Y SIN ENFERMEDAD MENTAL GRAVE}

\section{CONCLUSIONES}

Tras el análisis de los resultados obtenidos se puede concluir que existen diferencias entre las personas con y sin enfermedad mental grave en cuanto al conocimiento, disponibilidad y uso de las nuevas tecnologías de la información. Concretamente, las personas con enfermedad mental tienen menos conocimientos, disponen menos de TIC y las utilizan menos, que las personas sin enfermedad mental. Esto puede entenderse como una evidencia de la exclusión social que este colectivo sufre, ya que pese a tener interés en las nuevas tecnologías, no se les brinda la oportunidad de aprender a utilizarlas, ni acceder a ellas.

También se encontraron diferencias en las opiniones que ambos colectivos tienen sobre las nuevas tecnologías. En concreto, las personas sin enfermedad mental opinan de manera más desfavorable acerca de las mismas. Esto puede ser consecuencia de la falta de conocimiento, ya que tras participar en el taller, dicho grupo pasa a considerar las TIC de manera más positiva.

Para concluir, cabe mencionar que a la hora de interpretar los resultados que del presente estudio se desprenden, hay que tener precaución, ya que se ha llevado a cabo con una muestra pequeña. Se debe recordar que esta investigación tiene el objetivo de servir como estudio piloto a futuras investigaciones de mayor alcance. Es necesario un estudio longitudinal cuantitativo con muestras de mayor tamaño. También se necesitan futuros estudios cualitativos para comprender cómo el uso del ordenador y demás TIC contribuye a mejorar la calidad de vida de las personas con trastornos mentales.

En otras palabras, teniendo en cuenta que el uso de TIC forma parte de nuestras actividades cotidianas y las posibilidades que estos medios ofrecen, sería interesante continuar estudiando las características del uso y conocimientos que la población con enfermedad mental posee, y los posibles efectos, tanto positivos como negativos, que el uso de las nuevas tecnologías podría tener en la vida diaria de estas personas.

\section{REFERENCIAS}

Agencia de Evaluación de Tecnologías Sanitarias (AETS), Instituto de Salud Carlos III. (2009). Las tecnologías de la información y comunicación en salud mental (Telepsiquiatría). Madrid: Author. Recuperado el 23 de junio de 2014, de http://gesdoc.isciii.es/gesdoccontroller?action=download\&id=14/09/2012-b1333e3664

Bravo, M. F. (2007). Dimensiones de los procesos de inclusión/exclusión social de personas con enfermedad mental. Tesis de maestría no publicada, Universidad Nacional de Colombia, Bogotá D.C., Colombia. Recuperado el 23 de junio de 2014, de http://www.coloquiodiscapacidad. com/investigaciones/phocadownload/Salud-vida-y-funcionamiento-humano/bravo_2007.pdf

Huang, Y-h. \& Su, I-J. (2009). Does Health Related Quality of Life Differ between People with Chronic Mental IIIness Who Use Computers and Those Who Do Not? Universal Access in $\mathrm{HCl}$, 361-366.

Moya, A. M. (2009). Las nuevas tecnologías en la educación. Revista Digital Innovación y experiencias educativas, (24). Recuperado el 22 de mayo de 2014, de http://www.csi-csif.es/andalucia/modules/mod_ense/revista/pdf/Numero_24/ANTONIA_M_MOYA_1.pdf

Välimäk, M., Hätönen, H., Lahti, M., Kuosmanen, L., \& Adams, C. E. (2012). Information and communication technology in patient education and support for people with schizophrenia. Cochrane Database of Systematic Reviews, 10. Recuperado el 18 de junio de 2014, de http://www.update-software.com/BCP/WileyPDF/EN/CD007198.pdf 